Reprod. Nutr. Dévelop., 1987, 27 (1 B), 257-258.

\title{
Effets du pourcentage de concentré de la ration et du niveau d'ingestion sur la vitesse de passage dans le rumen de différents types d'aliments chez le mouton
}

\author{
C. PONCET (*), J. GONZALEZ ( $\left.{ }^{1}\right)$, Brigitte MICHALET-DOREAU (**) \\ (*) Laboratoire de la Digestion des Ruminants, \\ (**) Laboratoire des Aliments, \\ I.N.R.A., Theix 63122 Ceyrat, France.
}

Summary. Raising the proportions of hay in the diet (40 to $90 \%$ ) or the level of feed intake $\left(40\right.$ to $65 \mathrm{~g} \mathrm{DM} / \mathrm{kg}^{0.75}$ ) increased the fractional outflow rates of rare earth-treated chopped hay, soybean meal and dried lucerne in sheep. Feed outflow rates appeared to be determined more by the type of total diet and feeding level than by the nature of the feed itself.

Nous avons mesuré, chez le mouton, les variations de la vitesse de passage dans le rumen de trois aliments en fonction du pourcentage de concentrés dans la ration et du niveau d'ingestion.

Matériel et méthodes. Trois moutons adultes, munis d'une canule simple du duodénum, ont reçu en 8 repas égaux/jour successivement 3 rations composées de foin de dactyle haché et d'un aliment concentré aggloméré lorge : $40 \%$; maïs : $40 \%$; tourteau de soja : $20 \%$ ) en proportions variables : $90: 10 ; 70: 30$ et $40: 60$. Ces 3 rations ont été distribuées à 2 niveaux d'ingestion : $41 \mathrm{~g}$ $\mathrm{MS} / \mathrm{kg}^{0,75}$ et à volonté : $59 \mathrm{~g} \mathrm{MS} / \mathrm{kg}^{0,75}$ pour la ration à $30 \%$ de concentré et $66 \mathrm{~g} \mathrm{MS} / \mathrm{kg}^{0,75}$ pour la ration a $60 \%$ de concentré ; la ration à $90 \%$ de foin n'a été distribuée qu'au niveau restreint. Trois aliments - foin de dactyle haché (FD), tourteau de soja (TS) et luzerne déshydratée (LD) - après avoir été débarrassés de leurs constituants rapidement fermentescibles par lavage $\left(1 \mathrm{~h}-90^{\circ}\right)$ en présence de détergent, ont été marqués par immersion dans une solution de terre rare radioactive : Ytterbium-169 pour le soja et la luzerne, Cérium-141 pour le foin (Ellis et Beever, 1984). La vitesse de passage de ces aliments dans les estomacs a été mesurée en suivant l'évolution de la concentration des marqueurs dans le contenu duodénal pendant $72 \mathrm{~h}$ après leur distribution. La vitesse de passage dans le rumen a été calculée en utilisant le modèle de Grovum et Williams (1973). La signification statistique des différents facteurs de variation - ration, niveau d'ingestion, aliment - a été déterminée par analyse de variance.

Résultats et discussion. Les taux de renouvellement $n$ 'ont pas été significativement différents entre aliments 10,$055 ; 0,057$ et $0,058 . \mathrm{h}^{-1}$ respectivement pour FD, TS et LD) bien que sensiblement plus faibles pour le foin de la ration a $10 \%$ de concentré. Ce résultat, en accord avec les données antérieures (cf. Eliman et Orskov, 1983) montre en outre que les taux de renouvellement des deux constituants de la ration, foin haché et concentré, sont quasiment égaux. Ainsi, la vitesse de passage d'un aliment dans le rumen ne serait pas déterminée uniquement par ses caractéristiques physiques et biochimiques, mais par celles de l'ensemble de la ration; les différents composants d'une ration constitueraient

(1) Adresse : E.T.S. Ingenieros Agronomos - Dept. de Nutr. Anim. 28040 Madrid (Espagne). 
une masse fibreuse qui séquestre les fines particules et freine leur évacuation (Faichney, 1986). Ce filtre semble moins efficace avec la ration riche en fibre (90\% de foin) qui maintient dans le rumen une phase liquide abondante, bien différenciée et circulant facilement.

L'augmentation du pourcentage de concentré dans la ration a entraîné une diminution significative du taux de renouvellement, identique pour le foin et les aliments concentrés - de 0,059 à $0,043 \cdot \mathrm{h}^{-1}$ entre 10 et $60 \%$ de concentré au niveau restreint, de 0,069 à $0,058 \cdot \mathrm{h}^{-1}$ entre 30 et $60 \%$ de concentré, à volonté (moyenne des 3 aliments). Cette évolution a été plus régulière pour les concentrés que pour le foin dont le taux de renouvellement n'a diminué qu'au-delà de $30 \%$ de concentrés.

TABL. 1. - Effets du niveau d'ingestion et de la proportion de concentré dans la ration sur le taux de renouvellement $\left(\mathrm{h}^{-1}\right)$ dans le rumen de 3 aliments : foin de dactyle haché (FD), tourteau de soja (TS). luzerne déshydratée (LD). (Moyenne \pm écart-type des résultats obtenus sur 3 moutons).

\begin{tabular}{|c|c|c|c|c|c|c|}
\hline \multirow{2}{*}{$\frac{\begin{array}{c}\text { Niveau } \\
\text { d'ingestion }\end{array}}{\text { Aliment }}$} & \multicolumn{3}{|c|}{ Restreint $\left(40 \mathrm{~g} \mathrm{MS} / \mathrm{kg}^{0,75}\right.$ ) } & \multicolumn{3}{|c|}{ A volonté } \\
\hline & FD & TS & LD & FD & TS & LD \\
\hline \multicolumn{7}{|l|}{$\begin{array}{c}\% \\
\text { de concentré }\end{array}$} \\
\hline 10 & $0,054(0,011)$ & $0,060_{(0,018)}$ & $0,064_{(0,013)}$ & - & - & - \\
\hline 30 & $0,055_{(0,005)}$ & $0,054(0,018)$ & $0,048 \quad(0,004)$ & ${ }^{0,067}(0,012)$ & $0,066_{(0,015)}$ & $0,073(0,003)$ \\
\hline 60 & $0,042(0,003)$ & $0,047_{(0,006)}$ & $\begin{array}{r}0,045 \\
\langle 0,005\rangle\end{array}$ & $\begin{array}{r}0,056{ }_{(0,005)} \\
(0,0)\end{array}$ & $0,059_{(0,006)}$ & $\begin{array}{r}0,058 \quad(0,009) \\
\quad\end{array}$ \\
\hline
\end{tabular}

Signification statistique : Effet " aliment », NS ; effet " ration », $P<0,05$; Effet " niveau d'ingestion $», \mathrm{P}<0,05$.

L'augmentation du niveau d'ingestion s'est accompagné d'un accroissement du taux de renouvellement - de 0,049 à $0,063 \cdot \mathrm{h}^{-1}$ en moyenne pour les 2 rations - comparable à celui enregistré par Eliman et Orskov (1984).

Ainsi, le pourcentage de concentré et le niveau d'ingestion d'une ration modifient la vitesse de passage des aliments dans le rumen (de 14 à $23 \mathrm{~h}$ dans cet essai) et par conséquent leur dégradation à ce niveau. Cependant, ces mêmes facteurs ont un effet sur l'intensité de l'activité microbienne opposé à celui qu'ils ont sur la vitesse de passage (Gonzalez, Michalet-Doreau et Poncet, 1987). II faut donc prendre en compte l'ensemble de ces effets antagonistes pour ne pas surestimer l'influence du temps de séjour sur la dégradabilité d'un aliment dans le rumen. Enfin, la vitesse de passage d'un aliment semble dépendre principalement de la composition et du niveau d'ingestion de la ration dans laquelle il est inclus. Au plan pratique, ceci devrait permettre de limiter le nombre de mesures nécessaires pour caractériser la vitesse de passage des aliments, aux principaux types de rations utilisés et aux niveaux d'ingestion élevés.

Ellis W. C., Beever D. E., 1984. In P. M. Kennedy, Techniques in particule size analysis of feed and digesta in ruminants. Can. Soc. Anim. Sci., Edmonton, 167.

Eliman M. E., Orskov E. R., 1984. Anim. Prod., 39, 201-206.

Faichney G. J., 1986. Proc. V/e Int. Symp. on Ruminant Physiology.

Gonzalez J., Michalet-Doreau B., Poncet C., 1987. Reprod. Nutr. Dévelop., 27, 255-256.

Grovum W. L., Williams V. J., 1973. Brit. J. Nutr., 30, 313-329. 